\title{
Neuropathological diagnosis and CAG repeat expansion in Huntington's disease
}

\author{
J H Xuereb, J C MacMillan, R Snell, P Davies, P S Harper
}

\begin{abstract}
Objective-To correlate the degree of CAG repeat expansion with neuropathological findings in Huntington's disease.

Methods-The CAG repeat polymorphism was analysed in a large series of brain samples from 268 patients with a clinical diagnosis of Huntington's disease in which full neuropathological data was available.
\end{abstract}

Results-Analysis by polymerase chain reaction was successful in $63 \%$ of samples (169 of 268). Repeat expansions were detected in 152 of $153(99 \%)$ samples with a neuropathological diagnosis of Huntington's disease. The exceptional case (22 CAG repeats) showed mild but definite pathological changes and had a typical clinical illness with a positive family history; it raises the possibility that an alternative mutation in the Huntington's disease gene may be responsible although it is more likely that a mutation in another gene has resulted in an Huntington's disease-like phenotype. Four of 16 cases without pathological changes of Huntington's disease also possessed an expanded repeat sequence; a glioblastoma masked the pathological changes of Huntington's disease in one case but the other three cases had a typical clinical history and a positive family history. These three cases may reliably be classified as Vonsattel's Huntington's disease grade 0 . Three of 12 cases with a normal repeat length and no morphological changes of Huntington's disease showed other neuropathology; two had Alzheimer's disease and the other had multiple sclerosis. Review of the clinical notes of two other cases indicated a diagnosis of tardive dyskinesia complicating phenothiazine treatment of schizophrenia. The remaining pathology negative/ expansion negative cases had been referred because of a family history of Huntington's disease; all but one were themselves symptom free. Apart from emphasising the possibility that an alternative mutation may result in a clinical phenocopy of Huntington's disease, this case suggests that such a clinical phenocopy may occur without gross or light microscopical neuropathological changes (Vonsattel's Huntington's disease grade 0). Conclusions-The study confirms the value of molecular genetic analysis in cases of suspected Huntington's disease and shows the importance of detailed neuropathological study in the few cases of suspected Huntington's disease with normal CAG repeat lengths.

(F Neurol Neurosurg Psychiatry 1996;60:78-81)

Keywords: Huntington's disease; CAG repeat; brain pathology

The identification of characteristic brain pathology has, until recently, been the gold standard for the definitive diagnosis of Huntington's disease. The cloning of the Huntington's disease gene (IT15) ${ }^{1}$ has provided the opportunity to reassess the sensitivity and specificity of the neuropathological diagnosis of the disease. A preliminary report, examining a few cases (22) of neuropathologically confirmed Huntington's disease showed some correlation between the degree of repeat expansion and the severity of caudate atrophy. ${ }^{2}$ Here we report the results of the analysis of a larger series of cases of clinically diagnosed Huntington's disease and attempt to correlate the degree of repeat expansion with the neuropathological findings. The study gives some indication of the future role of necropsy in Huntington's disease.

\section{Method}

Frozen half brains (one cerebral hemisphere and attached half of brainstem and cerebellar hemisphere) from 296 cases of clinically diagnosed Huntington's disease were submitted to the Cambridge Brain Bank Laboratory between April 1981 and June 1993. The mean age at death was 58 (range 23-85) years; we do not have reliable data about duration of disease. The corresponding formalin fixed brain tissue from just under half the cases was examined neuropathologically by Dr L Carrasco at Runwell Hospital, Essex, UK. These cases largely antedated publication of the grading scheme of Vonsattel et al (Vonsattel's grade) ${ }^{3}$ for the severity of Huntington's disease. Most of the other cases were examined at the Cambridge Brain Bank Laboratory by one of us (JHX) and Huntington's disease grading ${ }^{3}$ was applied at the time of neuropathological examination. Pathological examination in the few remaining cases was undertaken in the original referring centre by an experienced neuropathologist. The neuropathological reports from all but 27 of the non-Cambridge cases were obtained 
Table 1 Neuropathological grading scale for Huntington's disease

\begin{tabular}{lll}
\hline Grade 0 & $\begin{array}{l}\text { Gross } \\
\text { Microscopical }\end{array}$ & $\begin{array}{l}\text { No abnormality } \\
\text { No abnormality }\end{array}$ \\
Grade 1 & Microscopical & $\begin{array}{l}\text { No abnormality } \\
\text { Cuadate: moderate gliosis } \\
\text { Putamen: mild gliosis }\end{array}$ \\
Gross & Microscopical & $\begin{array}{l}\text { Caudate: atrophy (convex medially) } \\
\text { Putamen: slight atrophy } \\
\text { Pallidus: normal } \\
\text { Caudate: moderate gliosis and neuronal loss } \\
\text { medially } \\
\text { Putamen: mild gliosis and neuronal loss dorsally }\end{array}$ \\
& Gross & $\begin{array}{l}\text { Caudate: atrophy (loss of medial convexity); } \\
\text { Putamen and pallidus: moderate atrophy } \\
\text { Caudate: gliosis and neuronal loss, severe medially } \\
\text { and moderate laterally } \\
\text { Putamen: gliosis and neuronal loss, severe dorsally } \\
\text { and moderate ventrally }\end{array}$ \\
Grade 4 & Gross & $\begin{array}{l}\text { Caudate, putamen, and pallidus: severely atrophic } \\
\text { (concave medially) } \\
\text { Caudate and putamen: very severe gliosis and } \\
\text { neuronal loss throughout } \\
\text { Accumbens: moderate gliosis }\end{array}$ \\
\hline
\end{tabular}

After Vonsattel et al $1985 .^{3}$

and in most cases it was possible to apply Vonsattel's grade (table 1) retrospectively (JHX). In all cases, the assessment was carried out blind to the clinical and family history.

DNA was extracted from frozen samples of cerebral neocortex by standard techniques. Amplification, by polymerase chain reaction (PCR), of the region of IT15 containing the CAG repeat polymorphism was carried out as described previously. ${ }^{4}$ Quality control in our own laboratory shows that the estimated repeat length is reproducible to within \pm 1 repeat on successive analyses. Telenius et $\mathrm{al}^{5}$ have recently reported that the degree of somatic mosaicism for the expansion is greatest in the basal ganglia and cerebral cortex. In

Plot of CAG repeat size v grade in pathologically established Huntington's disease

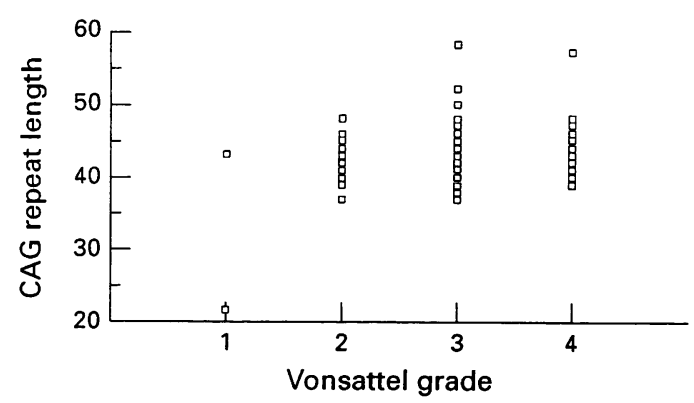

Table 216 cases with no pathological evidence of Huntington's disease (either grade 0 or not Huntington's disease)

\begin{tabular}{llllll}
\hline & Repeat length & $\begin{array}{l}\text { Choreiform } \\
\text { movements }\end{array}$ & Dementia & $\begin{array}{l}\text { Family } \\
\text { history }\end{array}$ & Neuropathology \\
\hline (CAG) $n \geqslant 37:$ & & & & Yes & Glioblastoma \\
A1 & 43 & Yes & No & Yes & Nil \\
A2 & 39 & Yes & No & Yes & Nil \\
A3 & 41 & Yes & Yes & Yes & Nil \\
A4 & 43 & Yes & No & Yes & \\
(CAG) $n<37:$ & & & & & \\
B1 & 17 & Yes & No & No & Nil \\
B2 & 21 & Yes & No & No & Nil \\
B3 & 21 & No & No & Yes & Nil \\
B4 & 23 & No & No & Yes & Nil \\
B5 & 21 & No & No & Yes & Nil \\
B6 & 22 & No & No & Yes & Nil \\
B7 & 22 & No & No & Yes & Nil \\
B8 & 29 & No & No & Yes & Nil \\
B9 & 22 & Yes & Yes & No & MS \\
B10 & 19 & No & Yes & No & AD \\
B11 & 36 & Yes & Yes & Yes & AD \\
B12 & 23 & Yes & Yes & Yes & Nil \\
\hline
\end{tabular}

our present study we merely noted the primary expansion size but we did not attempt to analyse the range of allele sizes amplified from each sample (we did not analyse the same cortical area in each case). In fact Telenius et al ${ }^{5}$ found that the predominant expanded allele did not change in size between tissues and McDonald et $a l^{6}$ found no evidence for somatic instability of the expanded allele. Statistical analyses ( $t$ tests and correlation coefficients) were carried out with the software package Cstat for Windows.

\section{Results}

Although neuropathological assessments were available on 268 of the original 296 cases (90\%) and Huntington's disease mutation results were successfully generated on 178 of the original $296(60 \%)$ frozen tissue samples, combined genetic and neuropathological data were available on only 169 cases $(57 \%)$.

One hundred and thirteen of these 169 cases were confidently allocated to one of Vonsattel's grades 1 to 4 . In 25 cases the changes were clearly at the severe end of the range but there was insufficient data to distinguish between grade 3 and 4 . In 15 cases there was no available record of the gross neuropathological findings to distinguish between grades 1-4, but a confident pathological diagnosis of Huntington's disease could be made on review of paraffin sections of basal ganglia and other brain areas.

The figure shows the relation between CAG repeat length and Vonsattel grade in the 113 cases of pathologically established Huntington's disease (excluding grade 0) which could be reliably graded. All the cases had an upper repeat length of 37 or over with one exception with a repeat length of 22 (pathologically grade 1). This case had a typical clinical history of choreiform movements and dementia together with a positive family history. There were no statistically significant differences ( $t$ test) between the mean repeat length for the cases in categories Huntington's disease grade $2-4$. There were too few cases (two) with Huntington's disease grade 1 to include this group in the analysis. One of these cases had a CAG repeat length of 43 and the other was normal. Likewise, there was no significant difference in the mean repeat length between those with mild changes (grades 1 and 2) and those grouped as severe pathological changes (grades 3 and 4 ).

Four of the 16 cases with no pathological evidence of Huntington's disease had repeat lengths in excess of 37 (table 2, cases A1-A4). One of these had a glioblastoma replacing the central white matter of the right parietal and occipital lobes. The putamen and caudate nucleus showed mild astrocytic hyperplasia but no noticeable nerve cell loss. This gliosis was considered secondary to glioblastomatous infiltration of the posterior limb of the internal capsule. The patient presented with a short history of "jitteriness of the left sided limbs" followed by blackouts. These were interpreted as simple partial motor seizures culminating in 
Table 3 Assessment of neuropathological diagnosis (test) of Huntington's disease

\begin{tabular}{lll}
\hline & \multicolumn{2}{l}{ Disease } \\
\cline { 2 - 3 } Test & Yes & No \\
\hline Positive & 153 & 0 \\
Negative & 4 & 11 \\
\hline Sensitivity $=\frac{153}{153+4} ;$ & specificity $=\frac{11}{0+11} ;$ predictive value $=$ \\
$\frac{153}{153+0}=100 \%$ positive and $=\frac{11}{4+11}=73 \%$ negative.
\end{tabular}

generalised seizure activity. Physical examination showed left sided sensory neglect and a left congruous homonymous hemianopia. Significantly, the admission notes record the presence of choreiform movements of the right sided limbs. Review of his brother's case notes indicate the appearance of "fidgetiness" and agitation at 37 years of age followed by the development of chorea and apathy. The clinical record of choreiform movements in the right sided limbs (ipsilateral, to the tumour), the positive family history, and the expanded triplet repeat show that the patient had Huntington's disease (grade 1) in addition to the cerebral tumour. The case highlights the diagnostic difficulty that may arise if only one cerebral hemisphere is available for morphological examination.

The other 3 expansion positive/morphology negative cases all had a long history of generalised choreiform movements and a positive family history, leaving no reasonable doubt about the diagnosis of Huntington's disease. These cases confirm the findings of Vonsattel et $a l^{3}$ that the disease may occur without gross or microscopical morphological changes in the basal ganglia. In this series, three of $169(1.8 \%)$ cases may be classified as Huntington's disease grade 0 .

Clinical review of the 12 expansion negative/morphology negative cases showed that in two cases (table 2, cases B1 and B2) the clinical diagnosis should be amended to tardive dyskinesia. Both patients had been diagnosed initially as schizophrenic and received phenothiazine treatment for many years before the onset of involuntary movements. In neither case was there a family history of chorea or dementia. Six expansion negative/morphology negative cases (table 2, cases B3-B8) were found on review not to have a history of involuntary movements or dementia. In each of these cases necropsy was prompted by the diagnosis of Huntington's disease in a relative and the need to reassure the subjects' own children.

There were three expansion negative/morphology negative (with regard to Huntington's disease) cases which showed alternative neuropathology. Severe chronic multiple sclerosis was present in one case (table 2, case B9) with a history of choreoathetosis and dementia; the mother had had "locomotor ataxia" but no necropsy was performed. The other two cases showed the characteristic changes of Alzheimer's disease. Case B10 (table 2) had never exhibited involuntary movements and the family history was negative. The clinical diagnosis of Huntington's disease had been suggested by a psychiatrist because "depression had been a significant component prior to the development of dementia." The clinical diagnosis of Huntington's disease could safely be revised in both these cases and triplet repeat sizes of 22 and 19 respectively were reassuring.

There remains uncertainty about the possible coincidence of Huntington's disease and Alzheimer's disease in case B11 (table 2). The clinical notes showed that involuntary movements had preceded the onset of dementia and there was a positive family history of Huntington's disease (necropsy had not been performed on deceased affected relatives). The histology of the basal ganglia was reviewed with knowledge of the clinical details of the case; mild astrocytic gliosis was noted in the dorsal part of the head of the caudate nucleus but the extent of this lesion remained insufficient for a confident pathological diagnosis of Huntington's disease. The genetic data were equally unclear, showing a triplet repeat size of 36 . Therefore, combined neuropathological and genetic tests failed to confirm or exclude Huntington's disease in one of 169 cases $(0.6 \%)$.

The remaining expansion-negative/morphology negative case (table 2, Case B12) had a typical clinical history of Huntington's disease as well as a positive family history of the condition. Necropsy showed no alternative neuropathology, except for mild Purkinje cell loss from the cerebellar cortex and acute ischaemic cell change in the hippocampal pyramidal cell layer which do not adequately explain the patient's clinical illness. Regrettably, there are no molecular data to confirm whether the other "affected" members of the family were mutation positive.

\section{Discussion}

This study shows the difficulty in successfully extracting DNA for PCR from stored brain tissue even when the tissue has been frozen rather than formalin fixed. Our laboratory uses standard techniques for the preparation of material for PCR analysis and the high failure rate for amplification is surprising. We are not aware of any data from other laboratories on the success of PCR amplification from stored brain tissue. It may be that the slow freezing process employed for these brains (very few were snap frozen) resulted in significant DNA degradation preventing successful PCR. Upadhyaya $e t a l^{7}$ reported degraded DNA in $66 \%$ of frozen brain samples subjected to Southern analysis and recommended that DNA be isolated from the tissue before storage. Clinicians and pathologists should be aware that even PCR analysis on stored brain tissue may not be possible in a large proportion of cases. This is important when discussing the need to confirm by DNA analysis a clinical/pathological diagnosis of Huntington's disease in a deceased relative of someone requesting presymptomatic testing.

This study confirms the sensitivity of neuropathological examination in the confirma- 
tion of the diagnosis of Huntington's disease. The disease was diagnosed pathologically in 153 out of 157 cases with expanded CAG repeats and a characteristic clinical course $(97.4 \%)$, supported in the vast majority of cases by a positive family history (table 3 ; case B11 with a borderline CAG repeat expansion is excluded from assessment of sensitivity). This study also allows an estimate of the specificity of neuropathological examination. In four cases regarded as neuropathologically negative for Huntington's disease at the initial blind assessment, the clinical diagnosis of Huntington's disease was confirmed by CAG repeat expansion, a positive family history of Huntington's disease, and the presence of characteristic choreiform movements. Excluding case B12 (which may be a clinical phenocopy of Huntington's disease), 11 neuropathologically Huntington's disease negative cases were all confirmed not to have the disease at review of clinical and genetic data, giving a $100 \%$ specificity. Therefore, an experienced neuropathologist is unlikely to make a false positive diagnosis of Huntington's disease but may expect to make a false negative diagnosis in $2.4 \%$ of cases in which the diagnosis of Huntington's disease is raised. The risk of a false negative diagnosis would be reduced if the entire brain was available for neuropathological examination but the known existence of "chemical" Huntington's disease (grade 0) would still be expected to lead to a false negative rate of $1.8 \%$ in the hands of a neuropathologist.

Although it is possible to assess the accuracy of neuropathological diagnosis on the basis of CAG repeat expansion, it is not possible to assess the screening performance of the molecular genetic test on the basis of neuropathological examination because an apparently false negative genetic result might be a rare Huntington's disease phenocopy. The singular case with neuropathological changes of Huntington's disease (grade 1), characteristic clinical course, positive family history, and a repeat length of 22 suggests that another, as yet undetected, mutation in the Huntington's gene or in a different gene may result in a neuropathological as well as clinical phenocopy of Huntington's disease. Another case (case B12, table 2) had a normal repeat length and no neuropathological changes despite a typical clinical illness and a positive family history, indicating the possibility that a Vonsattel's grade 0 phenocopy may occur. The rarity of such cases means that they would have little impact on the counselling of relatives of those with clinical Huntington's disease.

This study shows that neuropathological examination remains a valuable diagnostic procedure in clinically suspicious cases of Huntington's disease with a normal CAG repeat size. In such problematic cases, it is recommended that the entire brain is reserved for morphological study. Furthermore, neuropathological examination should be done blind to the clinical information in the first instance. Clinicians are often faced with the dilemma of whether subtle clinical signs are a disease manifestation or merely a reflection of an anxious patient (for example, mild impairment of rapid alternating hand movements). The knowledge that there is a positive family history of Huntington's disease can bias towards judging such a finding as significant. In their study of cases of Huntington's disease without repeat expansion, Andrew et al ${ }^{8}$ reported one case with a positive family history and pathological confirmation of the diagnosis but no repeat expansion (three other affected family members showed an expansion). They report that reassessment of the case clearly indicated that the pathological findings were, after all, not consistent with Huntington's disease. They noted that the positive family history may have influenced the first neuropathological assessment. In the present study, which was carried out blind to the family history and clinical evaluation, there was no false positive neuropathological diagnosis of Huntington's disease.

One particular case (B11, table 2) in this study indicates the difficulty which may arise when the CAG repeat size is neither clearly normal nor clearly abnormal. We suspect that this is a case of Huntington's disease-grade 0 with coincidental Alzheimer's disease because of the typical choreiform illness and positive family history. The Huntington's Disease Collaborative Research Group ${ }^{1}$ measured CAG repeat size in 173 normal subjects and 74 cases of Huntington's disease. All the patients with Huntington's disease had repeat lengths of 42 or greater and most normal samples (169 of 173) were 24 or less. Two normal samples were in the 25-30 range and two were in the 30-41 range. The study of Neal et $a l^{2}$ showed that pathologically established Huntington's disease can occur with a gene mutation which results in repeat lengths of 38 and our own series of pathologically established disease includes cases with repeat lengths of 37. Necropsy should be sought in cases with repeat lengths in the 30-36 range, even if there is a typical choreiform illness and a positive family history, to establish the lower limit of repeat size which may be associated with pathologically established Huntington's disease.

1 The Huntington's Disease Collaborative Research Group. A novel gene containing a trinucleotide repeat that is expanded and unstable on Huntington's disease chromosomes. Cell 1993;72:971-83.

2 Neal JW, Fenton I, Macmillan JC, et al. A study comparing mutation triplet repeat size and phenotypes in patients with Huntington's disease. Neurodegeneration 1994;3: 73-7.

3 Vonsattel JP, Myers RH, Stevens TJ, Ferrante RJ, Bird ED Richardson EP. Neuropathological classification of Huntington's disease. $\mathcal{F}$ Neuropathol Exp Neurol 1985;44: 559-77.

4 Snell R, MacMillan JC, Cheadle JP, et al. Relationship between trinucleotide repeat expansion and phenotypic variation in Huntington's disease. Nature Genetics 1993;4:393-7.

5 Telenius H, Kremer B, Goldberg YP, et al. Somatic and gonadal mosaicism of the Huntington disease gene repeat in brain and sperm. Nature Genetics 1994;6:409-14.

6 McDonald ME, Barnes G, Srinidhi J, et al. Gametic but not somatic instability of CAG repeat length in Huntington's disease. $\mathcal{F}$ Med Genetics 1993;30:982-6.

7 Upadhyaya M, Reynolds GP, Harper PS. Recombinant DNA studies on stored necropsy brain samples from patients with Huntington's chorea. $\mathcal{F}$ Clin Pathol 1985; 38:1093-5.

8 Andrew SE, Goldberg YP, Kremer B, et al. Huntington's disease without CAG expansion: phenocopies or errors in assignment? Am f Hum Genet 1994;54:852-63. 